\title{
Parámetros poblacionales de la jaiba azul Callinectes sapidus (Rathbun, 1896) en la bahía de Chetumal, Quintana Roo, México
}

\author{
Population parameters of the blue crab Callinectes sapidus (Rathbun, 1896) \\ in Chetumal Bay, Quintana Roo, Mexico
}

Carmen O. Rosas-Correa ${ }^{1}$ y Alberto de Jesús-Navarrete ${ }^{1}$

\author{
${ }^{1}$ Departamento de Aprovechamiento y Manejo de Recursos Acuáticos. El Colegio de la Frontera Sur Unidad Chetumal, Centenario \\ km 5.5, Chetumal, Quintana Roo, México. C.P. 77900 \\ callinectes2002@msn.com
}

\begin{abstract}
To evaluate the blue crab population state in the Chetumal Bay, independent and dependent samplings of the fishery were carried out, considering climatic seasons: coldfront, dry and rainy. A total of 2177 blue crabs were collected, 1146 coming from the fishery and 1031 from independent sampling. The size-frequency data showed two age groups, (60 to $119 \mathrm{~mm}$ and 120 to $179 \mathrm{~mm}$ carapace width). Higher crabs abundance occurred during the rainy season. Using only male dependent and independent data, the parameters of von Bertalanffy's model were obtained: $\mathrm{K}=0.51$ (year ${ }^{-1}$ ); $\mathrm{L}_{\infty}=$ $231.50 \mathrm{~mm} \mathrm{CW}$ and $\mathrm{t}_{0}=-0.11$. Total mortality, $\mathrm{Z}$, was 0.85 , whereas natural mortality, $\mathrm{M}$, was 0.66 , and fishing mortality, $\mathrm{F}$, was 0.19 . Based on the relation between the yield-per-recruit $\left(\mathrm{Y}^{\prime} / \mathrm{R}\right)$ and the average rate exploitation $\mathrm{E}$ was 0.22 . We conclude that the resource is not over-fished and can sustain the current level of exploitation Emax $=0.65$.
\end{abstract}

Key words: Fisheries, dependent and independent data, population
Resumen.- Para evaluar el estado de la población de la jaiba azul en la bahía de Chetumal, se tomaron datos dependientes e independientes de la pesquería, considerando tres temporadas climáticas: frentes fríos, temporada de secas y temporada de lluvias. Un total de 2177 jaibas fueron recolectadas, 1146 correspondieron a los datos de la pesquería y 1031 del muestreo independiente. La frecuencia de tallas mostró dos grupos de edad (60 a 119 mm y 120 a 179 mm, de ancho del caparazón). La mayor abundancia de jaiba azul ocurrió en la época de lluvias. Usando únicamente las tallas de las jaibas machos, de los datos dependientes e independientes, se obtuvieron los parámetros del modelo de von Bertalanffy: $\mathrm{K}=0,51$ (año ${ }^{-1}$ ); $\mathrm{L}_{\infty}=231,50 \mathrm{~mm} \mathrm{AC} \mathrm{y} \mathrm{t}_{0}=-0,11$. La mortalidad total, $\mathrm{Z}$, fue 0,85, sin embargo la mortalidad natural, $M$, fue 0,66, y la mortalidad por pesca $\mathrm{F}$ fue 0,19 . Basados en la relación del rendimiento por recluta ( $\left.\mathrm{Y}^{\prime} / \mathrm{R}\right)$ y el promedio de la tasa de explotación $\mathrm{E}$ fue 0,22. Concluimos que el recurso no está sobreexplotado y puede sostener el nivel actual de explotación Emax $=0,65$.

Palabras clave: Pesquerías, datos dependientes e independientes, población

\section{Introducción}

Las jaibas del género Callinectes sostienen pesquerías importantes en varias partes del mundo y tienen una alta demanda. Datos recientes de la captura mundial de la jaiba azul (Callinectes sapidus Rathbun, 1896) indican que en la última década los desembarcos han promediado alrededor de 110000 t (FAO 2000). Estados Unidos de América es uno de los principales consumidores y exportadores de jaiba capturándose a lo largo de la costa este y sur del Atlántico. La bahía de Chesapeake aporta la mayoría de la pesquería comercial y recreativa. En México, la jaiba es un recurso pesquero artesanal que se explota con mayor intensidad en el litoral del Pacífico, capturándose al menos 18 especies de jaibas y cangrejos, de las cuales 10 pertenecen al género Callinectes, mientras que la pesca en el Golfo y Caribe del país es menos intensa. En la parte centro-norte del Golfo de México, Heck et al. (2001) mencionaron la importancia de captura de jaiba al señalar que para 1987 se obtuvieron ganancias aproximadamente de US\$37 millones, ocupando el segundo lugar en importancia económica después del camarón (Litopenaeus spp.). En tanto que en el litoral del Golfo de México y Caribe, las capturas de jaiba durante el año 2002, ascendieron a 8,600 t (Medellín et al. 2003). En Yucatán y Quintana Roo esta pesquería está sustentada por organismos de talla y valor reducido, por lo que se considera como pesca alternativa. La captura en Yucatán presentó una producción máxima en 1990 de $370 \mathrm{t}$, la cual ha venido disminuyendo hasta llegar a $50 \mathrm{t}$. En Quintana Roo esta pesquería no se ha desarrollado completamente, y se reporta una captura máxima de $7 \mathrm{t}$ con un promedio anual de $1 \mathrm{t}$ (SAGARPA, 2006). En la 
bahía de Chetumal la pesquería de jaiba es una actividad relativamente reciente, pues los pescadores mencionan que hace 5 años capturaban jaiba en esta bahía. Aunque la captura de jaiba se realiza prácticamente todo el año, hay una temporada alta que va de mayo a septiembre. Actualmente para el recurso no existen medidas de regulación (veda, cuota y talla mínima de captura), y debido a los bajos costos de operación para su captura (aros jaiberos), la hacen susceptible de ser explotada sin control.

Por lo anterior, los objetivos de esta investigación fueron: 1) generar información básica sobre el estado de la población, 2) estimar los parámetros de crecimiento y 3) evaluar las tasas de mortalidad de esta especie. Finalmente, en base a la relación entre rendimiento por recluta y la tasa de explotación, se realiza una evaluación preliminar del recurso que servirá de referencia para realizar más investigaciones en la estimación de la población y su disponibilidad, así como para normar su aprovechamiento con criterios de sustentabilidad.

\section{Material y métodos}

El estudio se realizó en la bahía de Chetumal, localizada entre los paralelos $18^{\circ} 11^{\prime}$ y $18^{\circ} 52^{\prime} \mathrm{N}$ y $87^{\circ} 51^{\prime}$ y $88^{\circ} 23^{\prime}$ O. El área de estudio se dividió en celdas de un minuto geográfico por lado, agrupándose en tres sectores, cubriendo ambas costas y la parte central de la bahía de Chetumal. Se eligieron al azar 30 sitios de muestreo, ubicando 14 sitios en el sector A, ocho en el sector B y ocho en el sector C (Fig. 1). El trabajo de campo se llevó a cabo en el periodo enero - septiembre 2002. Se realizaron tres muestreos con una duración de nueve días cada uno e incluyeron las tres temporadas climáticas de la región: frentes fríos (enero-febrero), temporada de secas (mayo-junio) y temporada de lluvias (agostoseptiembre de 2002).

Los organismos se recolectaron con dos tipos de trampas cangrejeras, las primeras de estructura de alambrón y cubierta de tela de gallinero, con una abertura de malla de 2,54 cm, y con dimensiones de 45 x 45 x 40 $\mathrm{cm}$, las segundas cubiertas de malla palapera de $2,54 \mathrm{~cm}$ de luz de malla y con dimensiones de 100 x 50 x $30 \mathrm{~cm}$. En cada sitio de muestreo, se colocaron dos transectos paralelos a la línea de costa y con tres trampas cada uno, separadas $30 \mathrm{~m}$ una de otra. Las trampas se cebaron con carne de bagre y colocaron a partir de las 09:00 h, extrayendo su contenido al día siguiente, aproximadamente a la misma hora y repitiendo esta operación por 9 días. A los ejemplares recolectados se les midió el ancho del caparazón (AC, mm) de extremo a extremo de las espinas laterales con un vernier de $1 \mathrm{~mm}$

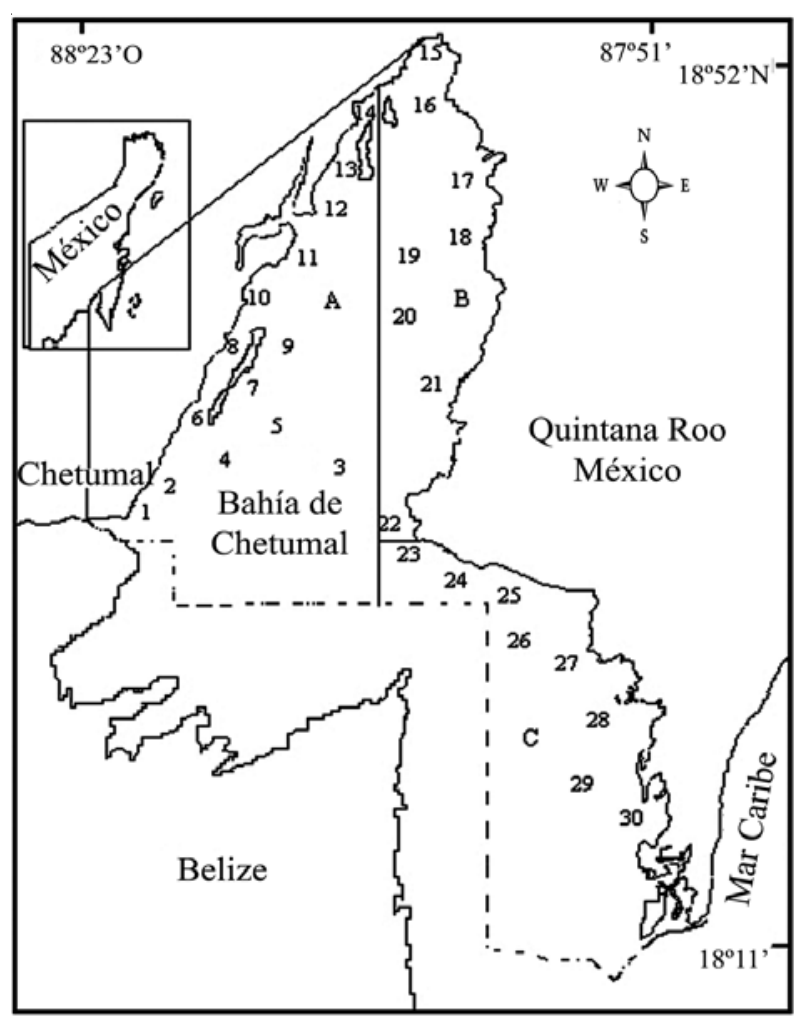

Figura 1

Localización del área de estudio y ubicación de los sitios de muestreo (Sectores A, B y C)

Location of the study area and sampling sites (Sectors A, B, and C)

en precisión. El sexo se determinó por la forma del abdomen, según lo señalado por Millikin \& Williams (1984).

Para determinar las diferencias entre las longitudes medias de la captura comercial y la captura independiente, se aplicó un ANOVA de dos vías, con el método de Tukey $(P<0,05)$. Utilizando el ancho del caparazón se elaboraron los histogramas de frecuencia relativa, de $10 \mathrm{~mm}$ para el tamaño de clase basado en la fórmula de Wolf (1989), usando solamente dos grupos modales en la muestra. De acuerdo con el criterio de Rugolo et al. (1998), el ancho del caparazón fue clasificado en grupos de edad (AC, $\mathrm{mm})$ : edad 0 (0-59 mm), edad 1 (60-119 mm) y edad 2 (120-179 mm).

Con la distribución de frecuencia de tamaño, se determinaron los parámetros de crecimiento de von Bertalanffy ( $\mathrm{L}_{\infty} \mathrm{y} \mathrm{K}$ ), utilizando el método indirecto ELEFAN I del programa FiSAT II (Gayanilo et al. 2001). El índice de crecimiento (Pauly \& Munro 1984) fue determinado para comparar los parámetros de crecimiento 
$\left(\mathrm{L}_{\infty}\right.$ (en $\left.\mathrm{cm}\right)$ y $\left.\mathrm{K}\right)$ de esta especie en diferentes sitios: Chesapeake, Delaware y Golfo de México. La mortalidad total se estimó por el método de Ehrhardt \& Ault (1992). La mortalidad natural se calculó con la ecuación propuesta por Pauly (1980) que considera que la mortalidad natural está correlacionada con la temperatura del ambiente. En este caso se utilizó la temperatura promedio de la bahía de Chetumal $\left(29^{\circ} \mathrm{C}\right)$ (Ortiz et al. 2007). La mortalidad por pesca (F) se obtuvo por la diferencia entre $\mathrm{Z}$ (mortalidad total) y M (mortalidad natural). La tasa media de explotación E, se calculó como la razón F / Z (Doi 1975). Se hizo una evaluación simple del recurso con base en la relación entre rendimiento por recluta relativo (Y’/ R) y la tasa media de explotación E (Pauly 1984). Esto requiere conocer $\mathrm{M}, \mathrm{K}$ (constante de crecimiento), $\mathrm{L}_{\infty}$ (longitud infinita), E y Lc (talla de primera captura); en este caso se tomó $\mathrm{Lc}_{c}=\mathrm{L}^{\prime}$ (punto medio del intervalo de talla que empieza a ser capturado).

\section{Resultados}

Un total de 2177 jaibas fue recolectado, 1146 correspondieron a los datos de la pesquería y 1031 del muestreo independiente (2058 machos y 119 hembras), lo que representa una proporción sexual de 18:1. No se encontraron diferencias significativas de las longitudes medias del caparazón entre los datos dependientes e independientes de la pesquería, (ANOVA de dos vías, Tukey $P<0,05$ ) por lo que se decidió trabajar con el total de datos para el análisis de la población.

De acuerdo con el criterio de Rugolo et al. (1998), la distribución de tamaños estuvo representada por dos grupos de edad, el primer grupo correspondió a jaibas entre 60 y 119 mm de AC y el segundo grupo correspondió a tallas entre 120 y 179 mm de AC. Los tamaños iguales o mayores a $180 \mathrm{~mm}$ fueron muy escasos $(0,4 \%$ del total de la población). Las frecuencias relativas del ancho del caparazón para las tres condiciones climáticas se muestran en la Fig. 2. La mayor frecuencia relativa se presentó en la temporada climática de lluvias. Las tallas más frecuentes encontradas en el punto medio fueron $135 \mathrm{y}$ 145 mm (Fig. 3).

Los parámetros de crecimiento se calcularon de la estructura de tallas global, dando como resultado una $\mathrm{L}_{\infty}=231,50 \mathrm{~mm}$ AC y una tasa de crecimiento $\mathrm{K}=0,51$ año ${ }^{-1}$. Los valores de $\varphi^{\prime}$ fueron similares entre la bahía de Chetumal y otros sitios dedicados a esta pesquería. La bahía de Chetumal registró un valor bajo de $\varphi^{\prime}(2,22)$ (Tabla 1).

Con la distribución de las frecuencias de longitudes total se utilizó una $\mathrm{L}=139,23$ mm y L' = 105 mm; este

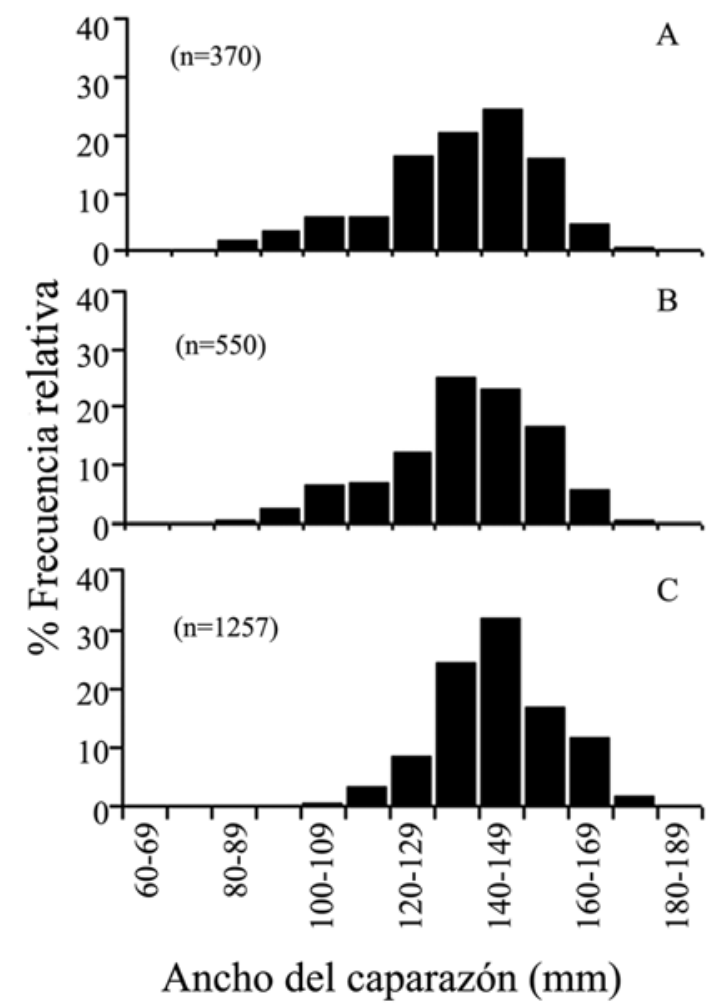

Figura 2

Frecuencia relativa del ancho del caparazón (AC, mm) de las clases de talla para $C$. sapidus en la bahía de Chetumal. A)

Estación fría, B) Estación seca y C) Estación lluviosa

Relative frequency of carapace width (AC, $\mathrm{mm}$ ) for C. sapidus in Chetumal Bay. A) cold season, B) dry season and $\mathrm{C}$ ) rainy season

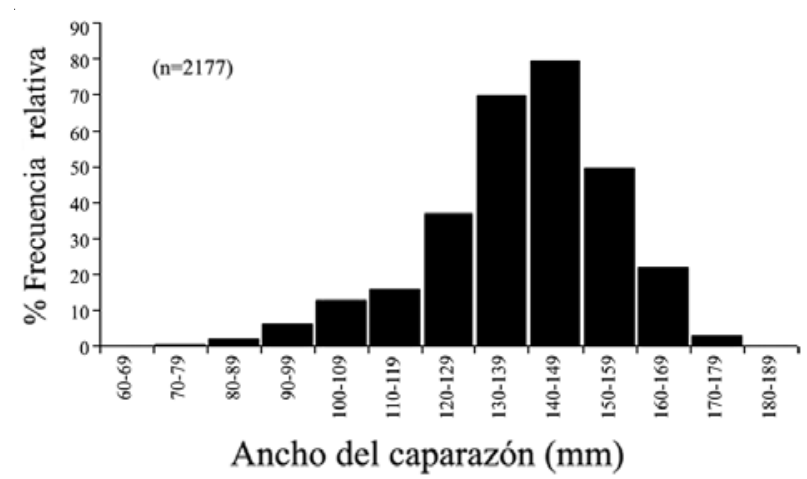

Figura 3

Distribución total de frecuencias de talla (AC, $\mathrm{mm})$ de $C$. sapidus en la bahía de Chetumal, Q. Roo para la temporada enero-septiembre 2002

Total size frequency distribution (AC, mm) for C. sapidus in Chetumal Bay, Q. Roo, data from January to September, 2002 
Tabla 1

Parámetros de crecimiento de $C$. sapidus $;^{1}=$ calculada $\mathrm{F}=\mathrm{Z}-\mathrm{M}$ para cada $\mathrm{M}, \mathrm{y}^{2}=$ estimada $\mathrm{E}$ para cada $\mathrm{F}$ con $\mathrm{Z}=0,85$

C. sapidus growth parameters; ${ }^{1}=$ calculated $\mathrm{F}=\mathrm{Z}-\mathrm{M}$ for each $\mathrm{M}$ and ${ }^{2}=\mathrm{E}$ estimated for each $\mathrm{F}$ at $\mathrm{Z}=0.85$

\begin{tabular}{|c|c|c|c|c|}
\hline & $\begin{array}{c}\text { Chesapeake } \\
\text { (Rugolo et al. 1998) }\end{array}$ & $\begin{array}{c}\text { Delaware } \\
\text { (Helser } \text { et al. 2001) }\end{array}$ & $\begin{array}{l}\text { Golfo de México } \\
\text { (Figaredo 1986) } \\
\text { (INP 2000) }\end{array}$ & $\begin{array}{l}\text { Bahía de Chetumal } \\
\text { (Este trabajo) }\end{array}$ \\
\hline \multicolumn{5}{|c|}{ Parámetros de crecimiento } \\
\hline $\mathrm{L}_{\infty}(\mathrm{mm})$ & 262,5 & 234,7 & 202,0 & 231,5 \\
\hline$K\left(\right.$ año $\left.{ }^{-1}\right)$ & 0,59 & 0,75 & 0,75 & 0,51 \\
\hline $\mathrm{t}_{0}$ & & & & $-0,11$ \\
\hline Índice $\varphi^{\prime}$ & 2,60 & 2,61 & 2,48 & 2,22 \\
\hline \multicolumn{5}{|c|}{ Tasas de mortalidad } \\
\hline Z & 1,55 & 1,17 & 3,91 & 0,85 \\
\hline M & 0,37 & 0,75 & 1,59 & 0,66 \\
\hline $\mathrm{F}$ & 1,18 & 0,42 & 2,32 & 0,19 \\
\hline & $0,48^{1}$ & $0,11^{1}$ & $-0,73^{1}$ & \\
\hline $\mathrm{F}_{\text {media }}$ & & & & 0,26 \\
\hline \multicolumn{5}{|c|}{ Tasas de explotación } \\
\hline \multirow[t]{2}{*}{ E } & 0,75 & 0,36 & 0,59 & 0,22 \\
\hline & $0,56^{2}$ & $0,12^{2}$ & $-0,84^{2}$ & \\
\hline $\mathrm{E}_{\text {media }}$ & & & & 0,30 \\
\hline
\end{tabular}

valor es el punto medio del intervalo de talla, ya que la población está completamente representada y los organismos de ese tamaño están totalmente reclutados y son susceptibles a la captura (Fig. 3). Se estimó una mortalidad total $\mathrm{Z}=0,85$ y la mortalidad natural $\mathrm{M}=$ 0,66 (equivale a un máximo de esperanza de vida $=4,5$ años). Al carecer de elementos para elegir un sólo valor representativo de $\mathrm{M}$, los cálculos de $\mathrm{F}$ se hicieron para cada una de las $\mathbf{M}$ de diferentes lugares (bahías de Chesapeake, Delaware y Chetumal). Se obtuvieron valores de F entre 0,11 y 0,48 . Sin embargo, como criterio práctico se consideró el valor de Chesapeake, Delaware y Chetumal; $\mathrm{F}_{\text {media }}=0,26$ como representativo (Tabla 1). La tasa media de explotación $\mathrm{E}=\mathrm{F} / \mathrm{Z}$, se calculó con $\mathrm{Z}=$ 0,85; para cada valor de $\mathrm{F}$ se obtuvo un rango de $\mathrm{E}$ de 0,12 a 0,56 . Para mejores resultados se obtuvo la tasa de explotación media: $\mathrm{E}_{\text {media }}=0,3$, sin tomar en cuenta $\mathrm{F}$ y $\mathrm{E}$ del Golfo de México, debido a que su tasa de explotación se encuentra por encima del óptimo y como resultado arroja valores negativos al calcular la mortalidad por pesca. Con la talla promedio de primera captura $\mathrm{L}_{\mathrm{c}}=\mathrm{L}^{\prime}=$ $105 \mathrm{~mm}$ AC, $\mathrm{K}=$ 0,51 y L $\mathrm{L}_{\infty}=231,50$ del ELEFAN I, y M = 0,66; obtuvimos la tasa de explotación. El valor observado de $\mathrm{E}_{\text {media }}=0,3$, cayó en el punto donde todavía no se produce el máximo rendimiento relativo por recluta $\mathrm{E}_{\max }=0,64$ (Fig. 4).

\section{Discusión}

Los resultados obtenidos muestran una ausencia de jaibas menores de $60 \mathrm{~mm}$ en la población. Esto se atribuye a la selectividad del arte de pesca empleado (trampas jaiberas) condicionadas por la abertura de malla. Los datos mostraron una dominancia de machos sobre las hembras, aparentemente relacionado con el ambiente y la biología de la especie. Los machos de $C$. sapidus tienden a permanecer en áreas de mas baja salinidad que las hembras (Ortiz et al. 2007). Hines et al. (1987) encontraron que hay una división de hábitat por sexo, donde la proporción de sexos de los organismos adultos difiere espacialmente con respecto al pico de apareamiento y migración de las hembras a zonas de mayor salinidad. Otros estudios realizados en la región, han reportado que $C$. sapidus ocurre con mayor frecuencia en época de lluvias (Andrade 1996, Ortiz et al. 2007), lo cual coincide con los resultados obtenidos en este trabajo.

Las regulaciones establecidas en otros países para el manejo del recurso se aplican de acuerdo a las características locales de la pesquería, regulando artes de pesca, tallas mínimas de captura y el establecimiento de vedas (Williams 1984). Ramírez \& Hernández (1988) citan que en el Diario Oficial del 18 de abril de 1974, se estableció una medida de $110 \mathrm{~mm}$, como ancho del caparazón mínimo de captura para las costas de México. 


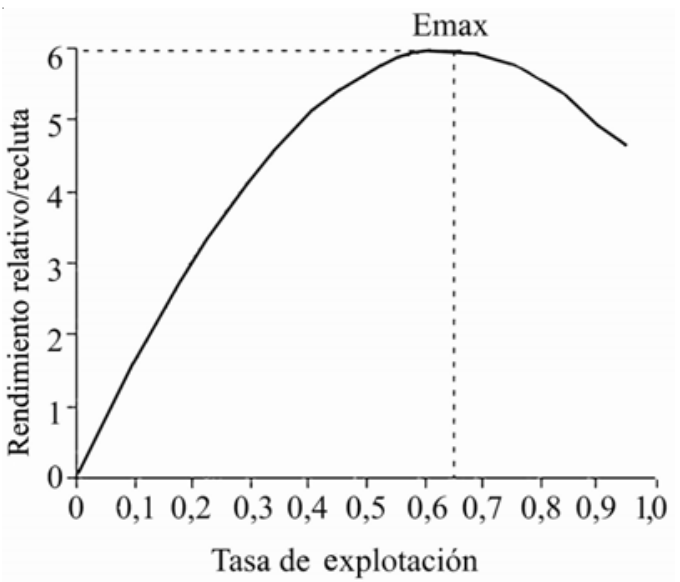

Figura 4

\section{Relación entre rendimiento relativo por recluta y tasa media de explotación en la pesquería de jaiba C. sapidus en la bahía de Chetumal, Q. Roo}

Relationship between relative yield per recruit and mean rate of exploitation in the $C$. sapidus crab fishery in Chetumal Bay, Q. Roo

En Tamaulipas se propuso desde 1987, una talla mínima legal de $140 \mathrm{~mm}$ de ancho del caparazón, debido a que la madurez gonádica de las jaibas está entre 110 y 130 mm (E. Arzate, en prep.). En Veracruz se recomendó manejar una talla mínima de $150 \mathrm{~mm}$, lo cual protegería al 55\% de las hembras sexualmente maduras (Loran et al. 1993). Las tallas mínimas de captura autorizadas en el Océano Pacífico incluido el Golfo de California son: de $120 \mathrm{~mm}$ de ancho del caparazón de las jaibas del género Callinectes (NOM-039-PESC-2003). En el presente estudio se registró una talla promedio de 139,23 mm AC que corresponde a organismos adultos, por lo que la talla mínima de captura mencionada anteriormente en el Diario Oficial, rebasa el ancho medio registrado para la población de $C$. sapidus en la bahía de Chetumal. Por tal motivo, se sugiere tomar en cuenta las características locales de la población y definir una talla para el adecuado manejo de la pesquería.

Los valores de $\varphi^{\prime}$ obtenidos en el trabajo fueron similares a los reportados en la literatura. El bajo valor registrado para la bahía de Chetumal, podría deberse a factores naturales que influyen, tales como temperatura y disponibilidad de alimento. $\mathrm{La}_{\infty}=231,5 \mathrm{~mm}$ (AC) de la bahía de Chetumal, es similar a la de la bahía Delaware $\mathrm{L}_{\infty}=234,7 \mathrm{~mm}$ (AC), pero menor a la de Chesapeake $(262,5 \mathrm{~mm})$ y mayor que la del Golfo de México (202 mm). Los diferentes trabajos relacionados con esta especie muestran un rango de valores de $\mathrm{K}$ que oscilan entre 0,59 a 0,75 ; sin embargo, nuestro valor fue de 0,51 (Tabla 1), valor más bajo a los obtenidos por otros autores. Sin embargo, la comparación es difícil porque son estimaciones a partir de distintos métodos, diferentes tamaños de muestra, modos de explotación y selectividad de los artes de pesca. La evaluación del crecimiento en los crustáceos siempre ha sido difícil por el proceso de muda. En el caso de las jaibas, no existe un procedimiento único para determinar la edad precisa de una jaiba a una talla determinada (Ju et al. 1999). Es difícil asignar la edad absoluta para una talla o determinar la edad máxima en la población. Rothschild et al. (1992) propusieron dos modelos para considerar el crecimiento de la jaiba durante su vida: 1) una afinidad continua usando los principios de von Bertalanffy y 2) una afinidad discreta incorporando las funciones basadas en datos por intermuda y el crecimiento por muda. En este estudio se aplicó el modelo de crecimiento continuo porque se carece de información para aplicar el modelo de crecimiento discreto.

Para la estimación de la mortalidad total $\mathrm{Z}=0,85$ se eligió la ecuación de Ehrhardt \& Ault (1992) debido a que es más robusta en especies tropicales de altas tasas de crecimiento y de vida relativamente corta. Además, que no sobreestima el valor de Z, como lo hace la ecuación de Beverton \& Holt (1956). Al comparar el resultado con los obtenidos por otros autores (Rugolo et al. 1998, INP 2000 y Helser et al. 2001), se observa que la mortalidad encontrada en este trabajo fue menor, esto muy probablemente se debe a que en esos lugares (bahía Chesapeake, Golfo de México y bahía Delaware) la actividad de pesca sobre esta especie es a gran escala (comercial y recreativa), aunada a un mayor número de pescadores, embarcaciones y trampas dedicadas a esta pesquería, ocasionando que la mortalidad sea mayor. En cambio, en la bahía de Chetumal, el nivel de explotación es menor ya que esta especie es capturada por 4-5 pescadores constantes y con 15-40 trampas máximo cada uno, ocasionando que la mortalidad sea muy baja (Tabla 1).

La $\quad M=0,66$ de la bahía de Chetumal está en el intervalo de 0,37 a 0,75 estimados en otras áreas (Rugolo et al. Helser \& Kahn 2001, Eggleston 2004). Este valor de $\mathrm{M}$ debe ser cautelado al originarse de una ecuación utilizada para peces y no para crustáceos. Las jaibas de la bahía de Chetumal alcanzan una longevidad de 4,5 años de acuerdo a la mortalidad natural estimada en este trabajo. La longevidad de las jaibas Callinectes spp. varía de 2 a más de 8 años (bahía Chesapeake, Rugolo et al. 
1998), sin embargo, es difícil asignar una longevidad exacta para crustáceos.

El rango de la mortalidad por pesca de 0,11 a 0,48 , está por debajo de la mortalidad natural, indicando que no hay una presión de pesca que se ejerce para esta especie y por lo tanto no afecta a la población. Estas estimaciones de F son tentativas pues no existe información histórica sobre el esfuerzo de pesca en la bahía de Chetumal (Tabla 1).

Con base en suposiciones sobre la dinámica de los recursos entre la mortalidad F = M, Gulland (1971) sugirió que el nivel de explotación óptimo $\left(\mathrm{E}_{\mathrm{opt}}\right)$ se alcanza cuando $\mathrm{E}=0,5$; esto significa mantener a la población en el nivel máximo de producción cuando ésta se está explotando en condiciones óptimas. En este caso, para la bahía de Chetumal, E se encuentra por debajo del nivel de explotación óptimo, lo que indica que esta población está sub-explotada. Si se compara con otros sitios (Tabla 1), la tasa de explotación se encuentra por encima del óptimo $\mathrm{E}=0,5$, con excepción de la bahía Delaware, donde la pesquería es explotada en niveles moderados y bajo regulación; sin embargo, no se recomienda aumentar el esfuerzo pesquero. Los resultados obtenidos de la pesquería de jaiba en el Golfo de México indican que la población se encuentra sobreexplotada al igual que en la bahía de Chesapeake. De acuerdo con la estructura de tallas de la temporada enero-septiembre 2002, a la tasa de explotación de la pesquería de jaiba en la bahía de Chetumal le corresponde un rendimiento por recluta relativo por debajo del valor máximo (Fig. 4). En consecuencia, para la bahía de Chetumal existe la posibilidad de aumentar el esfuerzo pesquero bajo un esquema controlado y regulado.

La situación actual de la pesquería de jaiba azul en la bahía de Chetumal es de tipo artesanal, por lo tanto es aconsejable implementar medidas de co-manejo y la formación de un comité de la pesquería entre los usuarios del recurso (pescadores), organizaciones no gubernamentales, academias, instituciones de investigación y autoridades para participar en las decisiones del manejo del recurso, así como vigilar la aplicación de las medidas tales como 'inspectores comunitarios', que pueden ser desarrollada por los propios pescadores. Es recomendable realizar muestreos y registros de captura a lo largo de varios años, con el fin de poder contar con datos representativos de la mayor parte de la población, así como estudios biológicos y ecológicos, para poder regular el recurso con criterios de sustentabilidad y siguiendo las estrategias adecuadas. También se recomienda conocer a los que participan en las diferentes áreas de captura, establecer una cuota anual y limitar la temporada de captura, respetar la talla mínima de 130 mm AC tanto de hembras como machos de la jaiba del género Callinectes (Defeo et al. 1999), establecer como únicos artes de pesca legales las trampas jaiberas, exigir la liberación de hembras ovígeras y juveniles, y proteger las zonas de reclutamiento.

\section{Agradecimientos}

A Héctor Ortiz, José Oliva y Daniel Robelo por su valiosa colaboración en el trabajo de campo. A Jacobo Schmitter, Alejandro Medina y Eloy Sosa, por sus comentarios al manuscrito. Los comentarios de dos evaluadores anónimos mejoraron sustancialmente el documento.

\section{Literatura citada}

Andrade M. 1996. Aspectos sobre biología y ecología de las jaibas del género Callinectes de la laguna 'Ría Celestún', Yucatán, México. Tesis de Maestría, Centro de Investigación y de Estudios Avanzados, Mérida, Yucatán, 95 pp.

Beverton RJH \& SJ Holt. 1956. A review methods for estimating mortality rates in exploited fish populations, with special reference to sources of bias in catch sampling. Rapports et procés-verbaux des Reunions Conseil Permanent International pour l'Exploration de la mer 44: 277-285.

Defeo O, A Andrade, R Pérez \& J Cabrera. 1999. Pautas para el manejo de la pesquería de jaiba y camarón en un área natural protegida: el caso de la Ría Celestún YucatánCampeche, 86 pp. Pronatura, Mérida, Yucatán.

Doi T. 1975. Análisis matemático de poblaciones pesqueras. Compendio para uso práctico, 95 pp. Instituto Nacional de la Pesca, México.

Eggleston DB \& EG Johnson. 2004. Population dynamics and stock assessment of the blue crab in North Carolina. Final report, 252 pp. North Carolina Fishery Resource Grant Program, North Carolina Sea Grant.

Ehrhardt NM \& JS Ault. 1992. Analysis of two length-based mortality models applied to bounded catch length frequencies. American Fisheries Society 121: 115-122.

FAO. 2000. Fishery Statistics: Capture Production, 713 pp. Food and Agriculture Organization, Rome.

Gayanilo FC, P Sparre \& P Pauly. 2001. FAO-ICLARM Stock Assessment Tools. Computerized Information, 119 pp. Food and Agriculture Organization of the United Nations, Rome.

Gulland JA. 1971. Manual de métodos para la evaluación de poblaciones de peces. 164 pp. Food and Agriculture Organization, Madrid.

Heck KLJr, LD Coen \& SG Morgan. 2001. Pre- and postsettlement factor as determinants of juvenile blue crab Callinectes sapidus abundance: results from the northcentral Gulf of Mexico. Marine Ecology Progress Series 222: 163-176. 
Helser TE \& DM Khan. 2001. Stock Assessment of Delaware Bay blue crab (Callinectes sapidus) for 2001. Department of Natural Resources and Environmental Control Delaware, 41 pp. Division of Fish and Wildlife, Dover, Delaware.

Hines AH, RN Lipcius \& M Haddon. 1987. Population dynamics and habitat partitioning by size, sex, and molt stage of blue crabs Callinectes sapidus in a subestuary of central Chesapeake Bay. Marine Ecology Progress Series 36: 55-64.

Instituto Nacional de la Pesca. 2000. La pesquería de jaiba del Golfo de México y mar Caribe. Carta Nacional Pesquera, 25 pp. Instituto Nacional de la Pesca, México.

Ju S, DH Secor \& HR Harvey. 1999. Use of extractable lipofuscin for age determination of the blue crab Callinectes sapidus. Marine Ecology Progress Series 185: 171-179.

Loran NR, AG Valdez \& GF Escudero. 1993. Algunos aspectos poblacionales de las jaibas Callinectes spp. en la laguna de Alvarado, Veracruz, México. Ciencia Pesquera 16: 15-31.

Medellín M, E Arzate, M Gómez \& A González. 2003. Memorias del II Foro Científico de Pesca Ribereña. 64 pp. SAGARPA- INP - CRIP Colima, México.

Millikin M \& A Williams. 1984. Synopsis of biological data on the blue crab Callinectes sapidus Rathbun. Food and Agriculture Organization Fisheries Synopsis 138: 51-131.

Norma Oficial Mexicana NOM-039-PESC-2003. 2006. Pesca responsable de jaiba en aguas de jurisdicción federal del litoral del Océano Pacífico. Diario Oficial de la Federación, México. Noviembre 11: 7-15.

Ortiz HJ, A de Jesús-Navarrete \& E Sosa. 2007. Distribución espacial y temporal del cangrejo Callinectes sapidus
(Decapoda: Portunidae) en la Bahía de Chetumal, Quintana Roo, México. Revista de Biología Tropical 551: 235-245.

Pauly D. 1980. On the interrelationships between natural mortality, growth parameters and mean environmental temperature in 175 fish stocks. Journal of Marine Science 39: $175-192$.

Pauly D. 1984. Fish population dynamics in tropical waters: a manual for use with programmable calculators, 325 pp. International Center for Living Aquatic Resources Management, Manila.

Pauly D \& JL Munro. 1984. Once more in the comparisons of the growth in fish and invertebrates. Fishbyte 2: 21.

Ramírez GM \& I Hernández. 1988. Investigación biológicopesquera para la obtención de jaiba suave Callinectes spp. en Alvarado, Veracruz. Tesis profesional, Universidad Nacional Autónoma de México, México, 35 pp.

Rothschild BJ, J Ault, E Vicent, S Smith, H Li, T Maurer, B Daugherty, G Davis, Ch Zhang \& R Garvey. 1992. Assessment of the Chesapeake Bay blue crab stock. Final report to the Maryland, 200 pp. Department of Natural Resources, The Chesapeake Bay Stock Assessment Committee, Maryland, Solomons, MD.

Rugolo LJ, KS Knotts, AM Lange \& VA Crecco. 1998. Stock assessment of Chesapeake Bay blue crab (Callinectes sapidus Rathbun). Journal of Shellfish Research 17: 906930.

Secretaría de Agricultura, Ganadería, Desarrollo Rural, Pesca \& Alimentación (SAGARPA). 2006. Diario Oficial de la Federación (Segunda Sección) Agosto 25: 29-30.

Williams AB 1984. The swimming crabs of the genus Callinectes (Decapoda: Portunidae). Fishery Bulletin 72: 685-798.

Recibido el 28 de noviembre de 2007 y aceptado el 5 de marzo de 2008 